\title{
Dynamic SPECT: evolution of a widely available tool for the assessment of coronary flow reserve
}

\author{
Simona Ben-Haim • Denis Agostini
}

Published online: 24 October 2014

(C) Springer-Verlag Berlin Heidelberg 2014

Myocardial perfusion imaging (MPI) SPECT is well established in the diagnosis, monitoring response to treatment and risk stratification in patients with known or suspected coronary artery disease (CAD). PET enables quantitative assessment of myocardial blood flow (MBF, in millilitres per gram per minute) and coronary flow reserve (CFR), and quantification with ${ }^{15} \mathrm{O}$-water, ${ }^{13} \mathrm{~N}$-ammonia and recently ${ }^{82} \mathrm{Rb}$ has been validated over a wide range of blood flows in animal models and humans [1-4]. Quantitative assessment of MBF has been shown to improve the diagnostic accuracy of conventional MPI with SPECT or PET, to improve cardiac risk assessment and to predict outcome [5-7]. Quantitation of MBF enables absolute assessment of myocardial flow and vasodilator reserve without the assumption of a normal reference region [8]. Therefore, the limitation of conventional MPI (underestimation of the extent and severity of multivessel $\mathrm{CAD}$, when tracer uptake in the best-perfused myocardial region does not represent normally perfused myocardium) can be overcome by the use of absolute quantitation [9].

Whereas PET is very costly and complex, SPECT systems are widely used for the assessment of myocardial perfusion in patients for the diagnosis and management of CAD. However, quantitative assessment with SPECT has been limited. To enable quantitation with SPECT a multidetector system is required to permit fast acquisition of dynamic data in

\section{S. Ben-Haim}

Institute of Nuclear Medicine, University College London,

University College Hospital, London, UK

\section{S. Ben-Haim}

Department of Nuclear Medicine, Chaim Sheba Medical Center,

Tel Hashomer, Israel

D. Agostini $(\bowtie)$

Department of Nuclear Medicine, EA 4650 Caen Normandy

University Hospital, CHU Côte de Nacre, Caen, France

e-mail: agostini-de@chu-caen.fr
$5-10 \mathrm{~s}$, and a suitable SPECT tracer is necessary. Transmission imaging for attenuation correction will allow accurate quantitation. Quantification of myocardial perfusion reserve has been attempted using SPECT and ${ }^{201} \mathrm{Tl}$ in dogs [10] and ${ }^{99 \mathrm{~m}}$ Tc-labelled tracers [10-12]. Dynamic SPECT imaging using multidetector SPECT systems and kinetic modelling of ${ }^{99 \mathrm{~m}} \mathrm{Tc}$-teboroxime has shown good correlation with microsphere-determined blood flow. However, limitations in detector sensitivity and temporal resolution of conventional SPECT systems prohibit further assessment $[10,11]$.

Another SPECT technique based on first-pass planar imaging followed by conventional SPECT MPI has been used to estimate a retention index of MBF and CFR [12]. This technique has shown a generally good correlation with PETmeasured flow, but CFR is underestimated at high flow rates [13]. The use of a retention index to estimate CFR using this method compared to absolute MBF from PET results in an underestimation of CFR values in the SPECT-based technique, since tracer retention decreases with increasing blood flow [14]. Spatial and timing resolution are poorer with SPECT and the tracer retention index underestimates CFR compared to quantitative PET. SPECT is indeed simpler than PET but this technique, unlike PET, does not include dynamic acquisition of tomographic data. In addition, the technique works only for tracers that act like microspheres, showing a constant extraction over a large range of flow rates and showing no washout from the time of injection to the time of measurement.

Conventional SPECT systems are limited in the dynamic collection of tomographic data. These systems consist of slowly rotating cameras with large detectors. The detectors' orbit is limited by mechanical as well as safety factors and the angular projections obtained are inconsistent, resulting in blurred images and possible bias in the estimated kinetic parameters. In addition, conventional detector crystals suffer 
from limited temporal resolution, and may not be able to collect adequate numbers of counts when tracer concentrations are rapidly changing, such as in dynamic acquisitions.

Recently introduced dedicated cardiac cameras with cadmium zinc telluride (CZT) crystals provide significantly improved sensitivity (eight to ten times better than conventional SPECT) and significantly improved resolution and energy resolution $[15,16]$. These improvements, in addition to allowing focused imaging of the heart and the use of new reconstruction algorithms, enable dynamic SPECT with kinetic analysis of ${ }^{99 \mathrm{~m}} \mathrm{Tc}-\mathrm{MIBI}$ myocardial concentrations to be performed in animals and humans [17-20], providing incremental diagnostic information over perfusion data alone [17]. Further studies are needed to assess whether availability of transmission to correct for attenuation may enable better modelling of ${ }^{99 \mathrm{~m}} \mathrm{Tc}$-labelled tracer kinetics. In addition, the new technology with superior sensitivity allows a significant reduction in the administered dose of ${ }^{99 \mathrm{~m}} \mathrm{Tc}$-labelled tracers [21-23], which should also be applied to dynamic SPECT in future studies.

With the increasing abundance of dedicated cardiac cameras with solid-state detectors, this technology offers great promise for CFR quantification with dynamic SPECT. However, further studies are needed for validation of this technique with PET measurements in large patient cohorts and with low administered activities, to establish its role in the clinical work-up of patients with known or suspected CAD and to provide data equivalent to the large body of data already accumulated for blood flow quantitation with PET tracers.

Acknowledgments UCL and UCLH are supported by the National Institute for Health Research, University College London Hospitals Biomedical Research Centre.

Conflicts of interest Dr Ben-Haim is advisor to Spectrum-Dynamics.

\section{References}

1. Hutchins GD, Schwaiger M, Rosenspire KC, Krivokapich J, Schelbert H, Kuhl DE. Noninvasive quantification of regional blood flow in the human heart using N-13 ammonia and dynamic positron emission tomography imaging. J Am Coll Cardiol. 1990;15:103242.

2. Lortie M, Beanlands RS, Yoshinaga K, Klein R, DaSilva JN, DeKemp RA. Quantification of myocardial blood flow with 82-Rb dynamic PET imaging. Eur J Nucl Med Mol Imaging. 2007;43: 1765-74.

3. El Fakhri G, Karan A, Sitek A, Dorbala S, Abi-Hatem N, Lahoud Y, et al. Reproducibility and accuracy of quantitative myocardial blood flow assessment with (82)Rb PET: comparison with N-13 ammonia PET. J Nucl Med. 2009;50:1062-71.

4. Tahari AK, Lee A, Rajaram M, Fukushima K, Lodge MA, Lee BC, et al. Absolute myocardial flow quantification with 82Rb PET-CT: comparison of different software packages and methods. Eur J Nucl Med Mol Imaging. 2014;41:126-35.
5. Murthy VL, Naya M, Foster CR, Hainer J, Gaber M, Di Carli G, et al. Improved cardiac risk assessment with noninvasive measures of coronary flow reserve. Circulation. 2011;124:2215-24.

6. Naya M, Murthy VL, Taqueti VR, Foster CR, Klein J, Garber M, et al. Preserved coronary flow reserve effectively excludes high-risk coronary artery disease on angiography. J Nucl Med. 2014;55:24855.

7. Ziadi MC, DeKemp RA, Williams KA, Guo A, Chow BJ, Renaud $\mathrm{JM}$, et al. Impaired myocardial flow reserve on rubidium-82 positron emission tomography imaging predicts adverse outcomes in patients assessed for myocardial ischemia. J Am Coll Cardiol. 2011;58:740 8.

8. Gould KL, Lipskomb K, Hamilton GW. Physiologic basis for assessing critical coronary stenosis. Instantaneous flow response and regional distribution during coronary hyperemia as measures of coronary flow reserve. Am J Cardiol. 1974;33:87-94.

9. Murthy VL, Di Carli MF. Non-invasive quantification of coronary vascular dysfunction for diagnosis and management of coronary artery disease. J Nucl Cardiol. 2012;19:1060-72.

10. Iida H, Eberl S, Kim KM, Tamura Y, Ono Y, Nakazawa M, et al. Absolute quantitation of myocardial blood flow with $201 \mathrm{Tl}$ and dynamic SPECT in canine: optimization and validation ok kinetic modeling. Eur J Nucl Med Mol Imaging. 2008;35:896-905.

11. Smith AM, Gullberg GT, Christian PE, Datz FL. Kinetic modelling of teboroxime using dynamic SPECT imaging of a canine model. J Nucl Med. 1994;35:484-95.

12. Di Bella EV, Ross SG, Kadrmas DJ, Khare HS, Christian PE, McJames S, et al. Compartmental modeling of technetium-99mlabeled teboroxime with dynamic single-photon emission computed tomography: comparison with static thallium-201 in a canine model. Invest Radiol. 2001;36:178-85.

13. Daniele S, Nappi C, Acampa W. Incremental prognostic value of coronary flow reserve assessed with single-photon emission computed tomography. J Nucl Cardiol. 2011;18:612-9.

14. Ito Y, Katoh C, Noriyasu K, Kuge Y, Furuyama H, Morita K, et al. Estimation of myocardial blood flow and myocardial flow reserve by 99mTc-sestamibi imaging: comparison with the results of O-15 H2O PET. Eur J Nucl Med Mol Imaging. 2003;30:281-7.

15. DeKemp RA, Trottier M, Beanlands RSB. Incremental prognostic value of coronary flow reserve assessed with single-photon emission computed tomography. J Nucl Cardiol. 2011;18:541-3.

16. Gambhir SS, Berman DS, Ziffer J, Nagler M, Sandler M, Patton J, et al. A novel high-sensitivity rapid-acquisition single-photon cardiac imaging camera. J Nucl Med. 2009;50:635-43.

17. Imbert L, Poussier S, Franken PR, Songy B, Verger A, Morel O, et al. Compared performance of high-sensitivity cameras dedicated to myocardial perfusion SPECT: a comprehensive analysis of phantom and human images. J Nucl Med. 2012;53:1897-903.

18. Verger A, Djaballah W, Fourquet N, Rouzet F, Koehl G, Imbert L, et al. Comparison between stress myocardial perfusion SPECT recorded with cadmium-zinc-telluride and Anger cameras in various study protocols. Eur J Nucl Med Mol Imaging. 2013;40:331-40.

19. Verger A, Imbert L, Yagdigul Y, Fay R, Djaballah W, Rouzet F, et al. Factors affecting the myocardial activity acquired during exercise SPECT with a high-sensitivity cardiac CZT camera as compared with conventional Anger camera. Eur J Nucl Med Mol Imaging. 2014;41: $552-28$.

20. Ben-Haim S, Murthy VL, Breault C, Allie R, Sitek A, Roth N, et al. Quantification of myocardial perfusion reserve using dynamic SPECT imaging in humans: a feasibility study. J Nucl Med. 2013;54:873-9.

21. Wells RG, Timmins R, Klein R, Lockwood J, Marvin B, deKemp RA, et al. Dynamic SPECT measurement of absolute myocardial blood flow in porcine model. J Nucl Med. 2014. doi: 10.2967/ jnumed.114.139782 
22. Gimelli A, Bottai M, Giorgetti A, Genovesi D, Filidei E, Marzullo P. Evaluation of ischaemia in obese patients: feasibility and accuracy of a low-dose protocol with a cadmiumzinc telluride camera. Eur J Nucl Med Mol Imaging. 2012;39: 1254-61.
23. Einstein AJ, Blankstein R, Andrews H, Fish M, Padgett R, Hayes $\mathrm{SW}$, et al. Comparison of image quality, myocardial perfusion and left ventricular function between standard imaging and single-injection ultra-low-dose imaging using a high-efficiency SPECT camera: the MILLISIEVERT study. J Nucl Med. 2014;55:1430-7. 